\title{
Characterisation of patients with primary biliary cirrhosis responding to long term ursodeoxycholic acid treatment
}

M Leuschner, C F Dietrich, T You, C Seidl, J Raedle, G Herrmann, H Ackermann, U Leuschner

\begin{abstract}
Background-In some patients with primary biliary cirrhosis, ursodeoxycholic acid causes full biochemical normalisation of laboratory data; in others, indexes improve but do not become normal.

Aims-To characterise complete and incomplete responders.

Methods-Seventy patients with primary biliary cirrhosis were treated with ursodeoxycholic acid 10-15 mg/kg/day and followed up for 6-13 years.

Results-In 23 patients (33\%) with mainly stage I or II disease, cholestasis indexes and aminotransferases normalised within 1-5 years, except for antimitochondrial antibodies. Histological findings improved. Indexes were not normalised in 47 patients $(67 \%)$ although the improvement of their biochemical functions parallelled the trend in the first group. In these incomplete responders histological findings improved to a lesser extent. The only difference between the two groups before treatment was higher levels of alkaline phosphatase and $\gamma$ glutamyl transpeptidase in the incomplete responders. At onset of treatment the discriminant value separating responders from incomplete responders was $660 \mathrm{U} / 1$ for alkaline phosphatase and $131 \mathrm{U} / 1$ for $\gamma$ glutamyl transpeptidase. One year later it was 239 and $27 \mathrm{U} / 1$ (overall predictive value for responders $92 \%$, for incomplete responders $81 \%$ ). There were no differences between the two groups concerning immune status, antimitochondrial antibody subtypes, liver histology, or any other data. HLA-B39, DRB1*08, DQB1*04 dominated in both groups.

Conclusions-In patients with mainly early stages of primary biliary cirrhosis, higher values of alkaline phosphatase and $\gamma$ glutamyl transpeptidase are the only biochemical indexes which allow discrimination between patients who will completely or incompletely respond to ursodeoxycholic acid treatment. (Gut 2000;46:121-126)
\end{abstract}

Keywords: primary biliary cirrhosis; prognostic indexes; full response to ursodeoxycholic acid; incomplete responders; anti-p53 autoantibodies; HLA typing

Ursodeoxoycholic acid (UDCA) improves many laboratory indexes and liver histology in patients with primary biliary cirrhosis (PBC) and prolongs time free of transplantation. ${ }^{1-7}$ Whether UDCA improves symptoms is controversial. As some patients experience complete normalisation of all laboratory parameters and improvement of liver histology we investigated whether the pretreatment data of full responders differ from those of incomplete responders. Differences between the two groups might be able to predict which patients are suitable for treatment and might thus help to improve medical treatment.

A recently described variant of PBC with positive or negative antimitochondrial antibodies (AMA), positive antinuclear antibodies (ANA) or smooth muscle antibodies (SMA), and histological features of both PBC and chronic autoimmune hepatitis, is called overlap syndrome..$^{8-11}$ In our study there were only a few patients with overlap syndrome who responded to UDCA treatment like patients without the syndrome; it was not necessary therefore to differentiate between the two groups.

\section{Methods}

STUDY DESIGN

The study is a retrospective review of the data of 76 patients with PBC who were treated with ursodeoxycholic acid (Ursofalk, Cholit-Ursan; daily dosage $10-15 \mathrm{mg} / \mathrm{kg}$ body weight). Evaluation comprises patients treated during the years 1985 to 1992 and followed up for 6-13 years. Exclusion criteria were ascites, oesophageal varices, encephalopathy, need for liver transplantation, features suggestive of other liver diseases, or additional liver medications (glucocorticoids, azathioprine, colchicine, D-penicillamine, cyclosporin, chlorambucil). Six of the 76 patients with one or more of the exclusion criteria were not entered into the study. Because they were followed in a special outpatient clinic for PBC patients, none of the 70 remaining patients dropped out. Compliance to treatment was assessed by serum bile acid analysis.

Abbreviations used in this paper: AFT, $\alpha_{1}$ fetoprotein; ALT, alanine aminotransferase; AMA, antimitochondrial antibodies; ANA, antinuclear antibodies; AP, alkaline phosphatase; AST, aspartate aminotransferase; AT III, antithrombin III; CA 19-9, carbohydrate antigen 19-9; CHE, cholinesterase; GGT, $\gamma$ glutamyl transpeptidase; GLDH, glutamate dehydrogenase; $\mathrm{HBsAg}$, hepatitis B surface antigen; HCV, hepatitis C virus; PBC, primary biliary cirrhosis; SMA, smooth muscle antibodies; UDCA, ursodeoxycholic acid. 
The interval between first diagnosis and onset of therapy, and the duration of therapy were documented. The number of pregnancies, deliveries, and the duration and mode of administration of hormone preparations were registered. Pruritus, fatigue, joint pain, and unspecific abdominal complaints as well as concomitant diseases were assessed and graded according to a point score system $(0$, none; 1 , mild; 2 , moderate; 3 , severe; 4 , massive).

To exclude the development of ascites or signs of portosystemic shunts as indexes of disease progression during the treatment period of at least six years, all patients underwent ultrasonography every six to 12 months (V 328 Acuson 128, Siemens, Erlangen, Germany).

Before onset of therapy, endoscopic retrograde cholangiopancreaticography had been performed in all patients to exclude biliary obstruction. To exclude oesophageal varices all patients underwent gastroscopy initially and then every 12 months.

Laboratory investigations were performed by standard methods in serum unless stated otherwise: serum alkaline phosphatase (AP), $\gamma$ glutamyl transpeptidase (GGT), bilirubin, glutamate dehydrogenase $(\mathrm{GLDH})$, aspartate aminotransferase (AST), alanine aminotransferase (ALT), prothrombin time, albumin, cholinesterase (CHE), gammaglobulins, IgM, IgG, IgA, AMA and AMA-subtypes 2, 4, 8, 9 (ELISA, CF), ANA, SMA, carcinoembryonic antigen, $\alpha_{1}$ fetoprotein (AFP), and carbohydrate antigen 19-9 (CA 19-9). Total and individual bile acids were assessed by HPLC, as described previously. ${ }^{12}$

Anti-p53 autoantibodies were detected using an ELISA (Dianova, Hamburg, Germany) with solid phase recombinant wild type p53 protein. HLA class I antigens were defined by the standard microlymphocytotoxicity test procedure using peripheral lymphocytes. ${ }^{13}$ HLA typing of HLA DRB1 and DQB1 alleles was performed by polymerase chain reaction (PCR) amplification of genomic DNA using sequence specific primers (PCR-SSP). Genomic DNA was extracted from whole blood samples following the salting out procedure. ${ }^{14}$ Primer sequences and PCR reaction conditions were chosen as described elsewhere. ${ }^{15}$ Testing for anti-HCV (second generation), $\mathrm{HBsAg}$, and anti-HBc were performed using commercially available ELISAs, radioimmunoassays, and reverse transcriptase PCR (RTPCR).

Most investigations were performed repeatedly before onset of therapy and at eight week intervals thereafter. Bile acid analysis was done

Table 1 Entry data of patients ( $n=70$ ) responding fully or incompletely to ursodeoxycholic acid

\begin{tabular}{lll}
\hline Demography & Full responders & Incomplete responders \\
\hline Number & $23(32.9 \%)$ & $47(67.1 \%)$ \\
Sex (female:male) & $21(91.3 \%): 2(8.7 \%)$ & $42(89.4 \%): 5(10.6 \%)$ \\
Age at first diagnosis (y) & $44.6(11.9), 21-66$ & $45.3(9.4), 27-68$ \\
Age at start of treatment (y) & $50.7(9.1), 32-70$ & $49.9(9.5), 28-69$ \\
Total disease duration (y) & $10.7(8.0), 2-19$ & $10.5(5.3), 0.5-14$ \\
Time between diagnosis and start of treatment (y) & $6.0(7.0), 0-23$ & $4.6(4.2), 0-15$ \\
Time until normalisation (months) & $31.3(20.4), 1-132$ & - \\
Total treatment time (months) & $56.6(45.3), 6-157$ & $71.1(41.1), 10-162$ \\
\hline
\end{tabular}

Results expressed as mean (SD), range. once before therapy and then at two year intervals and at study end. The determination of AMA subtypes (PA Berg, Immunologisches Labor, Eberhard-Karls-Universität, Tübingen, Germany) was performed twice before therapy and again after 1-4 years; hepatitis serology was performed before therapy and 1-2 years later. HLA typing and determination of anti-p53 were done at the beginning of the study.

All patients had a liver biopsy before onset of therapy and at two year intervals thereafter. The staging was done according to the Ludwig criteria $^{16}$ : stage I, portal hepatitis; stage II, periportal hepatitis; stage III, fibrosis and bridging necrosis; stage IV, liver cirrhosis. The extent of inflammation, bile duct proliferation, bile duct destruction, bile duct paucity, granulomas, piecemeal necrosis, bridging necrosis, and contents of connective tissue were assessed according to a point score system ( 0 , none; 1 , mild; 2 , moderate; 3 , severe; 4 , massive). The evaluation of liver specimens was done by one pathologist without knowledge of the clinical data.

\section{STATISTICS}

Clinical data were expressed as mean (SD) and when appropriate as median values with limits of $50 \%$ interquartile ranges. For all tests the level of significance was set at 0.05 . Probability values were tested by the Wilcoxon-MannWhitney U test. For analysis of liver histology the Mantel-Haenszel test was used. The number of patients and controls positive for an HLA allele were compared by the $\chi^{2}$ test. Fisher's exact test (two tailed) was used when appropriate ( $n<5$ in either group).

As a control group we used 351 healthy blood donors (all controls were typed for HLA $\mathrm{A}, \mathrm{B}$, and $\mathrm{C}$ antigens and 270 were typed for HLA DRB1 and DQB1 alleles). Concerning the HLA analysis, $p$ values $\left(p_{n c}\right)$ were corrected $\left(p_{c}\right)$ for the number of comparisons (Bonferroni adjustment). The strength of an association was estimated by the relative risk (RR; Woolf's method). For positive (negative) association the aetiological (preventive) fraction was calculated. ${ }^{17}{ }^{18}$ Fisher's exact test was also used for differentiation of stages of the disease and of AMA subtypes. Computing a "prognostic index" in order to predict whether a patient presumably will be a responder or a partial responder, non-parametric discriminant analysis was applied.

\section{Results}

HISTOLOGICAL CLASSIFICATION OF PATIENTS WITH PBC

Twenty seven patients had stage I, 31 stage II, seven stage III, and five stage IV disease (early stages I and II: $82.9 \%$; stage III and stage IV without ascites, oesophageal varices, encephalopathy: $17.1 \%$ ).

FULL RESPONDERS: COMPLETE BIOCHEMICAL RESPONSE

Table 1 presents entry demographic data of patients. Twenty of the 23 responders $(87 \%)$ had PBC stage I or II, three (13\%) stage III or 
Table 2 Entry data and data after normalisation in full responders

\begin{tabular}{llll}
\hline Characteristic & At entry & After normalisation & $p$ Value \\
\hline $\begin{array}{l}\text { Cholestasis } \\
\text { Alkaline phosphatase (U/1) }\end{array}$ & $382(221), 148-1220$ & $129(30), 87-177$ & $\mathrm{p}<0.0001$ \\
$\begin{array}{l}\gamma \text { glutamyl transpeptidase (U/1) } \\
\text { Bilirubin (mg/l) }\end{array}$ & $208(199), 21-948$ & $18.1(15.3), 6-28$ & $\mathrm{p}<0.0001$ \\
$\begin{array}{l}\text { Inflammation } \\
\text { Glutamate dehydrogenase (U/1) }\end{array}$ & $15(40), 4-20$ & $5(2), 2-12$ & $\mathrm{p}<0.001$ \\
$\begin{array}{l}\text { Alanine aminotransferase (U/1) } \\
\text { Aspartate aminotransferase (U/1) }\end{array}$ & $19.7(13.9), 3-56$ & $1.7(1.0), 0.8-1.4$ & $\mathrm{p}<0.0001$ \\
$\begin{array}{l}\text { Immunology } \\
\text { IgM (mg/1) }\end{array}$ & $50.4(130.0), 16-560$ & $10.0(3.1), 6-17$ & $\mathrm{p}<0.0001$ \\
IgG (mg/l) & $53.5(99.1), 9-480$ & $9.1(2.2), 6-18$ & $\mathrm{p}<0.0001$ \\
Antimitochondiral antibodies & $6970(4450), 1710-20990$ & $3030(1630), 1330-2710$ & $\mathrm{p}<0.0001$ \\
& $17170(4280), 10480-26700$ & $13870(2870), 8470-21110$ & $\mathrm{p}<0.001$
\end{tabular}

Results expressed as mean (SD), range.

Normal reference ranges: <190 U/1 for alkaline phosphatase; 4-28 U/1 for $\gamma$ glutamyl transpeptidase; $<20 \mathrm{mg} / 1$ for bilirubin; $<4.5$ $\mathrm{U} / 1$ for glutamate dehydrogenase; $4-22 \mathrm{U} / 1$ for alanine aminotransferase; $6-18 \mathrm{U} / 1$ for aspartate aminotransferase; $600-2800 \mathrm{mg} / 1$ for IgM; and $8000-18000 \mathrm{mg} / \mathrm{l}$ for IgG.

IV. Before diagnosis of PBC there had been 19 deliveries of girls and 19 of boys (1:1), and one stillbirth. Eight women $(34.8 \%)$ had taken contraceptives for a short period (26 (7) months).

In 23 patients (33\%) all biochemical laboratory indexes normalised, except titres of AMA. Table 2 presents the most relevant laboratory findings of the 23 responders before therapy and after normalisation; figs $1,2,3$, and 4 show the course of the disease. The cholestastic enzymes normalised within 3-5 years, inflammation markers within 1-4 years, and $\operatorname{IgM}$ after five years of therapy. Within the first treatment year $50-80 \%$ of the overall improvement was achieved. In the following three years the indexes decreased less steeply until normalisation.

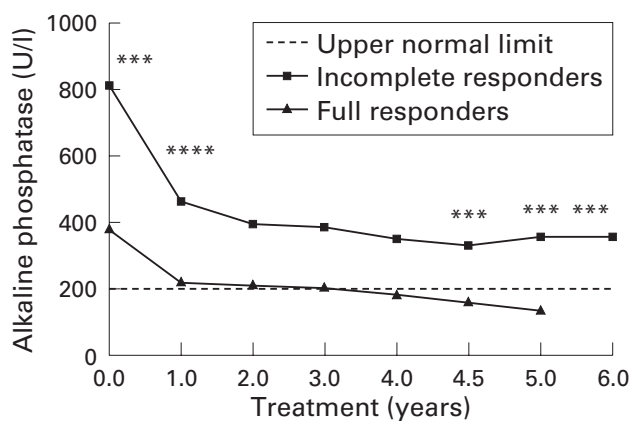

Figure 1 Alkaline phosphatase concentrations during ursodeoxycholic acid (UDCA) treatment. ${ }^{* *} p<0.001$, $\star \star \star * p<0.0001$ (since incomplete responders were treated for a longer period of time, the six year value was compared with the five year value of full responders).

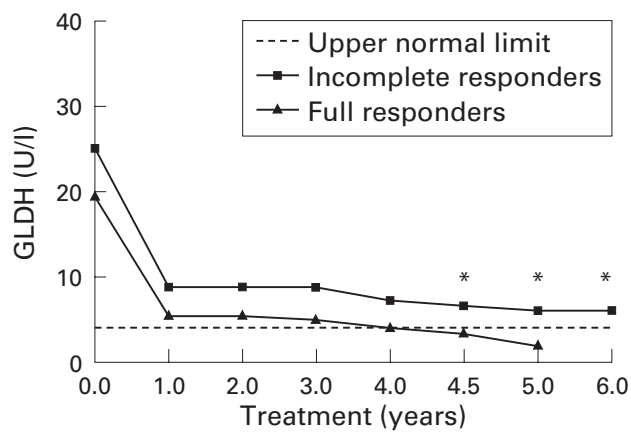

Figure 2 Glutamate dehydrogenase (GLDH) concentrations during ursodeoxycholic acid (UDCA) treatment. ${ }^{*} p<0.05$ (since incomplete responders were treated for a longer period of time, the six year value was compared with the five year value of full responders).
Table 3 presents immunological characteristics of full responders. AMA, ANA, and SMA remained unchanged. Ten patients had AMA subtypes $M_{2,4}$, five had $M_{2,4,9}$, four $M_{2,4,8}$, three $M_{2,8}$, and one $M_{2,9}$. The spectra did not change during therapy.

All other laboratory findings, such as lipase, AFP, and CA 19-9 were within normal range. Total cholesterol was mildly elevated in nine patients (maximum $2350 \mathrm{mg} / 1$; normal: less than $2000 \mathrm{mg} / \mathrm{l}$ ) and did not decrease consistently. The p53 index was between -0.157 and -0.252 (normal $f_{\mathrm{I}}<0$ ). Bile acid analysis showed that UDCA had increased in all patients to a mean of $42.3 \%$ of total bile acids in the serum. Hepatitis markers B (anti-HBs, anti-HBc) were found in one patient (4.3\%).

Analysis of HLA class I and class II antigens revealed a marginally increased frequency of $B$

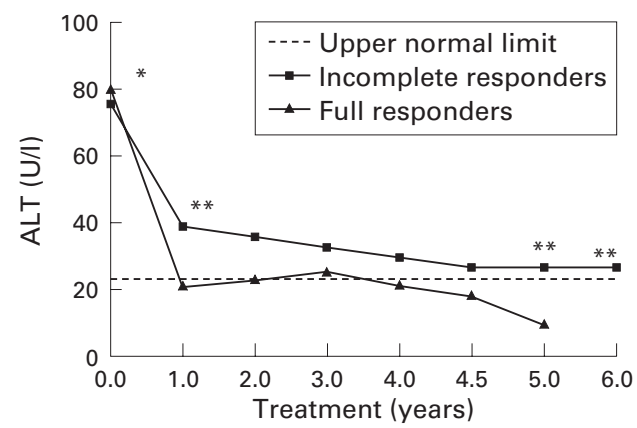

Figure 3 Alanine aminotransferase (ALT) concentrations during ursodeoxycholic acid (UDCA) treatment. ${ }^{\star} p<0.05$, ${ }_{*}^{*} p<0.01$ (since incomplete responders were treated for a longer period of time, the six year value was compared with the five year value of full responders).

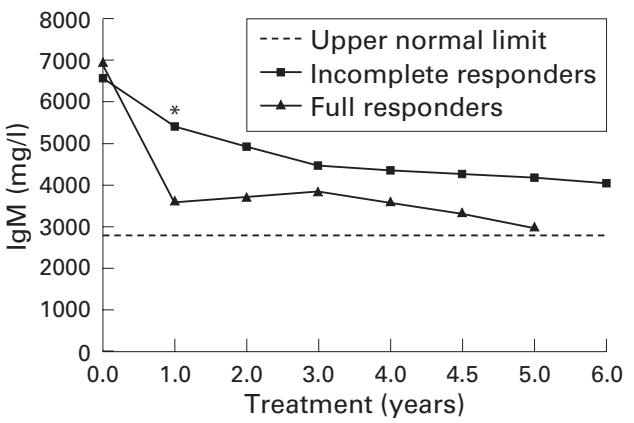

Figure 4 IgM levels during ursodeoxycholic acid (UDCA) treatment. ${ }^{*} p<0.05$ (since incomplete responders were treated for a longer period of time, the six year value was compared with the five year value of full responders). 
Table 3 Immunological characteristics of full and incomplete responders at entry

\begin{tabular}{lll}
\hline & Full responders $(n=23)$ & Incomplete responders $(n=47)$ \\
\hline $\begin{array}{ll}\text { Immunoglobulins mean }(S D), \text { range } \\
\text { IgM }\end{array}$ & $6970(4450), 1710-20990$ & $6600(3930), 1000-17200$ \\
IgG $^{\star}$ & $17170(4280), 10480-26700$ & $16790(4280), 10900-31600$ \\
Autoantibodies & & \\
AMA & $8 / 23(34.8 \%)$ & $17 / 47(36.2 \%)$ \\
AMA, ANA & $8 / 23(34.8 \%)$ & $12 / 47(25.5 \%)$ \\
AMA, ANA, SMA & $5 / 23(21.7 \%)$ & $12 / 47(25.5 \%)$ \\
AMA, SMA & $2 / 23(8.7 \%)$ & $6 / 47(12.8 \%)$ \\
\hline
\end{tabular}

^Normal reference ranges: $600-2800 \mathrm{mg} / 1$ for IgM; $8000-18000 \mathrm{mg} / 1$ for IgG.

alleles among patients with PBC in comparison to 351 healthy controls: HLA B39 (seven $(11.7 \%)$ versus eight (2.3\%), $\mathrm{RR}=5.66$, $\left.\mathrm{EF}=0.1, \mathrm{p}_{\mathrm{nc}}<0.001, \mathrm{p}_{\mathrm{c}}=\mathrm{NS}\right) ; \mathrm{HLA} \mathrm{DRB} 1^{\star} 08$ (eight $(13.3 \%)$ versus $14(5.2 \%), \mathrm{RR}=2.81$, $\left.\mathrm{EF}=0.09, \mathrm{p}_{\mathrm{nc}}<0.05, \mathrm{p}_{\mathrm{c}}=\mathrm{NS}\right) ;$ and $\mathrm{DQB} 1^{\star} 04$ (eight $(13.3 \%)$ versus nine $(3.5 \%), R R=4.21$, $\left.\mathrm{EF}=0.1, \mathrm{p}_{\mathrm{nc}}<0.01, \mathrm{p}_{\mathrm{c}}=\mathrm{NS}\right)$. These HLA alleles are in strong linkage disequilibrium. The number of patients, however, was too small to perform two locus haplotype analysis.

Liver histology after normalisation of liver biochemistry had improved notably. Over an average of five years the point score per patient had decreased from 2.14 to $1.34(\mathrm{p}<0.05)$.

Twenty two of 23 patients $(95.7 \%)$ reported complaints, most frequently fatigue $(52 \%)$, pruritus (44\%), and joint pain (44\%) followed by uncharacteristic abdominal discomfort $(13 \%)$. Abdominal discomfort disappeared in $100 \%$ of the patients, pruritus in $80 \%$, fatigue in $75 \%$, and joint pain in $63 \%$. Concomitant signs and diseases were found in $8 / 23$ patients $(34.8 \%)$ once each: erythema nodosum, psoriasis vulgaris, collagenous colitis, severe chronic polyarthritis, goitre with autonomous adenoma, arthritis, autoimmune thrombocytopenia, and CREST syndrome.

INCOMPLETE RESPONDERS: INCOMPLETE

BIOCHEMICAL RESPONSE

Before onset of therapy, the only difference between the 47 patients with incomplete response (67\%) and the $23(33 \%)$ with complete response was significantly higher AP $(\mathrm{p}<0.001)$ and GGT $(\mathrm{p}<0.03)($ table 4 , fig 1$)$. There were no differences in demographic data (table 1), the stage of the disease, number of births (boys:girls 1:1.2), administration of hormone preparations $(15 / 47=31.9 \%)$, inflammatory indexes (indexes even less in incomplete responders; table 4, figs 2 and 3), immune status (table 3, fig 4), HLA typing (see earlier), anti-p53 status, and liver histology. The figures show the course of the disease of incomplete responders. As in full responders a rapid improvement occurred within the first treatment year; thereafter they improved slightly, in parallel to the full responders, but indexes did not reach the normal range over the six years of follow up. Although the histological stages and the activity of the disease were not different in patients fully and incompletely responding to UDCA treatment, the point score in incomplete responders did not improve significantly (point score per patient before therapy: 2.13, and at evaluation: $1.94 ; \mathrm{NS}$ ).

Sixteen patients had AMA subtypes $\mathrm{M}_{2}, 15$ $M_{2,4}, 12 M_{2,4,8}$, and one patient each had $M_{4,8}$, $\mathrm{M}_{4}, \mathrm{M}_{9}$, and $\mathrm{M}_{2,4,9}$. The difference between full and incomplete responders was not significant $(p=0.17)$. Total and individual serum bile acid analysis during UDCA treatment showed no difference between full and incomplete responders: in neither group was there a significant increase in the more apolar bile acids chenodeoxycholic acid, deoxycholic acid, or lithocholic acid. Hepatitis B and C markers (anti-HBs, anti-HBc in three patients, anti$\mathrm{HCV}$ in one patient; RT-PCR excluded active disease) were found in incomplete responders only in four patients $(8.5 \%)$.

Forty four of 47 incomplete responders (93.6\%) reported complaints: fatigue $(63 \%)$, pruritus $(52 \%)$, joint pain $(21 \%)$, and uncharacteristic abdominal discomfort (4\%). Pruritus improved in $46 \%$ (disappeared in $6.8 \%$ ), fatigue improved or disappeared in $28 \%$, abdominal discomfort in $25 \%$, and joint pain in $22 \%$. Although assessment of symptoms is rather difficult and could have been influenced by the knowledge of whether biochemical data had improved or deteriorated, the influence of UDCA seemed to be more impressive in fully responding patients. Concomitant diseases were present in 17 of 47 patients (36.2\%): goitre with autonomous adenoma (two patients), hyperthyroidism, sicca syndrome (three patients), diabetes mellitus (three patients), severe osteoporosis (three patients), and one case each of fracture of a vertebral body, pulmonary fibrosis, Raynaud's disease, neurodermitis, von Willebrand syndrome, and CREST syndrome.

DISCRIMINANT ANALYSIS TO PREDICT RESPONSE In order to establish a prognostic decision index the laboratory data from before onset of therapy and after one year were analysed by means of non-parametric discriminant analysis. The derived prognostic rule was as follows:

Table 4 Differences between entry data of full responders and incomplete responders

\begin{tabular}{llll}
\hline Characteristic & Full responders $(n=23)$ & Incomplete responders $(n=47)$ & $p$ Value \\
\hline $\begin{array}{l}\text { Cholestasis } \\
\text { Alkaline phosphatase (U/1) }\end{array}$ & $382(221), 148-1220$ & $813(455), 199-1933$ & $\mathrm{p}<0.001$ \\
$\begin{array}{l}\gamma \text { glutamyl transpeptidase (U/1) } \\
\text { Bilirubin (mg/l) }\end{array}$ & $208(199), 21-948$ & $324(274), 53-1260$ & $\mathrm{p}<0.03$ \\
$\begin{array}{l}\text { Inflammation } \\
\text { Glutamate dehydrogenase (U/1) }\end{array}$ & $15(40), 4-200$ & $11(17), 4-120$ & $\mathrm{p}=0.2$ \\
$\begin{array}{l}\text { Alanine aminotransferase (U/1) } \\
\text { Aspartate aminotransferase (U/1) }\end{array}$ & $20(14), 3-56$ & $25(15), 5-63$ & $\mathrm{p}<0.05$ \\
\end{tabular}

Results expressed as mean (SD), range.

Normal reference ranges: $<190 \mathrm{U} / 1$ for alkaline phosphatase; $4-28 \mathrm{U} / 1$ for $\gamma$ glutamyl transpeptidase; $<20 \mathrm{mg} / 1$ for bilirubin; $<4.5$ $\mathrm{U} / 1$ for glutamate dehydrogenase; $4-22 \mathrm{U} / 1$ for alanine aminotransferase; $6-18 \mathrm{U} / 1$ for aspartate aminotransferase; 600-2800 mg/1 for IgM; and $8000-18000 \mathrm{mg} / \mathrm{l}$ for IgG. 
a patient with both AP less than 660 and GGT less than $131 \mathrm{U} / 1$ before onset of therapy is presumably a full responder (predictive value for full responders $52 \%$, for incomplete responders $78 \%$ ). After one year of treatment patients with both AP less than 239 and GGT less than $27 \mathrm{U} / 1$ are supposed to be full responders (predictive value for full responders $92 \%$, for incomplete responders $81 \%$ ).

OUTCOME

In responders the average duration of the disease until evaluation of data was 10.5 years. The average treatment time was 4.6 years. In one female patient we observed progression of PBC from histological stage II to stage III. In all patients liver function (prothrombin time, AT III, albumin, CHE) remained normal. Ascites or oesophageal varices did not develop. None of the patients had to undergo liver transplantation. One patient became pregnant after 3.5 years of therapy; in another patient amenorrhoea disappeared, having existed for several years. One patient died from a subarachnoid haemorrhage after 12 years of therapy. None of the full responders developed carcinoma.

In patients incompletely responding to therapy, the liver disease averaged 9.9 years; treatment lasted for an average of 5.9 years. In five of the 47 patients $(10.6 \%)$ the disease progressed: in three patients from stage II to III, and in one patient each from I to II and from I to IV. Only in one of the three patients with progression from II to III did liver function (prothrombin time, AT III, albumin, CHE) remain normal. One of the two developed mild splenomegaly; another developed grade 1 oesophageal varices. In the one patient with progression from stage I to II all other findings remained normal. The patient with progression from stage I to IV developed grade 2 oesophageal varices, splenomegaly, and reduced pulmonary function, but there was no change in liver function. None of the patients had to undergo liver transplantation. In four of the 47 incomplete responders five carcinomas developed (8.5\%): one each in the rectum, urinary bladder, or breast, and two in the cervix uteri.

\section{Discussion}

Our study in patients with mainly early stages of PBC has shown cholestasis indexes to be the most important prognostic markers of the response to UDCA treatment. This confirms data of an earlier two year study with late stages of the disease and much more severe elevations of AST, AP, and bilirubin. ${ }^{19} \mathrm{~A}$ retrospective evaluation of a large study with 548 patients corroborated this observation. ${ }^{720}$

In our study all 70 patients biochemically improved on therapy. Among them were 23 patients $(33 \%)$ in whom all laboratory findings, except AMA, normalised within 1-5 years (full responders) and liver histology improved notably. Because assessment of symptoms is difficult we refrained from calculating statistical significances, but the point score improved notably compared with incomplete responders.
One patient even became pregnant, and in another patient long standing secondary amenorrhoea disappeared. The remaining $47 \mathrm{pa}-$ tients $(67 \%)$ responded incompletely. In these incomplete responders histology improved to a lesser extent and progressed by one to three stages in $10 \%$. This was the case despite having a $23 \%$ shorter duration of disease before treatment and a $21 \%$ longer treatment period than the full responders.

A detailed analysis of a large number of indexes revealed that it is mainly higher initial levels of AP and GGT that distinguish incomplete responders from full responders. A trend to later stages of the disease in the group of incomplete responders was not significant statistically $(p=0.52)$. The comparison of biochemical parameters characterising the inflammation (even less in incomplete responders), the immune status, and all other data investigated, including data on a specific genetic predisposition, were of no value in predicting a therapeutic effect. The same was true of the histological score and presence of anti-p53 autoantibodies as a marker of cell proliferation, which may be overexpressed in non-tumorous liver cells of patients with liver cirrhosis. ${ }^{21}{ }^{22}$ Patients with the AMA profiles $M_{2,4}$ or $M_{2,4,8}$ were evenly distributed in both groups. This is of interest because it has been suggested previously that the subtype $\mathrm{M}_{4}$ in particular heralds a rather progressive course of the disease. ${ }^{23} 24$ The Mayo Clinic risk factor ${ }^{25} 26$ and the European risk factor ${ }^{27}$ did not permit a prediction of a drug response and the outcome.

If the course of cholestasis and inflammation (figs 1,2, and 3) is analysed, it is noteworthy that the laboratory indexes of full and incomplete responders parallel each other over most of the study period. Indexes improved rapidly during the first treatment year and only slightly from year 1 to 4 ; whereas indexes of full responders improved thereafter into the normal range, the indexes of incomplete responders levelled off and remained above the normal range. As shown by discriminant analyses, patients with AP values below 239 and a GGT below $27 \mathrm{U} / 1$ after one year of UDCA treatment have a $91 \%$ likelihood of full normalisation within the following two or three years. By contrast those with higher values of AP and GGT have an $81 \%$ likelihood of persisting abnormal biochemical indexes after four more years of treatment. The predictive value of the pretreatment biochemical indexes was less satisfactory.

Parallelism of the curves and the observation that the levels of alkaline phosphatase (and to a lesser extent of GGT) represent the only difference between full and incomplete responders suggest that one of the mechanisms by which ursodeoxycholic acid improves primary biliary cirrhosis could be its influence on bile secretion. The rate of improvement in cholestatic indexes is similar in the two groups, but the incomplete responders do not normalise because they start from higher initial values. In patients with mild cholestasis the ability of UDCA to induce choleresis appears to be sufficient to "cure" the disease biochemically. In 
those with more severe cholestasis, possibly more powerful choleretic compounds might be more efficacious. In these patients who responded incompletely to the UDCA regimen used in our study we have begun a prospective study of this question using a more powerful choleretic substance than UDCA. First results seem to support our hypothesis.

These considerations do not preclude the hypothesis that membrane stabilisation, ${ }^{28}{ }^{29}$ the stimulation of exocytosis, ${ }^{30}$ the protection of mitochondrial oxidative metabolism, ${ }^{31}{ }^{32}$ or the promotion of antioxidant activities ${ }^{33}$ might be additional effects of UDCA treatment due to the shift from hydrophobic to less toxic hydrophilic bile acids within the liver cell. ${ }^{34}$ However, as increasing the hydrophilicity of the circulating bile acid pool by administration of cholylsarcosine $^{35}$ to patients who had responded incompletely to UDCA did not further improve serum hepatic chemistry, we propose that it is the influence on cholestasis, rather than on hydrophobicity, by which UDCA exerts its beneficial effect in primary biliary cirrhosis.

1 David R, Kurtz W, Strohm WD, et al. Die Wirkung von Ursodesoxycholsäure bei chronischen Leberkrankheiten. Eine Pilotstudie [abstract]. Z Gastroenterol 1985;23:420.

2 Leuschner U, Fischer H, Kurtz W, et al. Ursodeoxycholic acid in primary biliary cirrhosis: results of a controlled double-blind trial. Gastroenterology 1989;97:1268-74

3 Heathcote EJL, Cauch-Dudek K, Walker V, et al. The Canadian multicentre double blind randomized controlled trial of ursodeoxycholic acid in primary biliary cirrhosis. Hepatology 1994;19:1149-56.

4 Lindor KD, Dickson ER, Baldus WP, et al. Ursodeoxycholic acid in the treatment of primary biliary cirrhosis. Gastroenterology 1994;106:1284-90.

5 Combes B, Carithers RL, Maddrey WC, et al. A randomized, double-blind, placebo-controlled trial of ursodeoxycholic acid in primary biliary cirrhosis. Hepatology 1995;22:759-66.

6 Leuschner U, Güldütuna S, Imhof M, et al. Effects of ursodeoxycholic acid after 4 to 12 years of therapy in early and late stages of primary biliary cirrhosis. F Hepatol 1994;21: late stag

7 Poupon RE, Lindor KD, Cauch-Dudek K, et al. Combined Poupon RE, Lindor KD, Cauch-Dudek K, et al. Combined acid in primary biliary cirrhosis. Gastroenterology 1997;113: acid in prin

8 Ben Ari Z, Dhillon AP, Sherlock S. Autoimmune cholangiopathy: part of the spectrum of autoimmune chronic active hepatitis. Hepatology 1993;18:10-15.

9 Michieletti P, Wanless IR, Katz A, et al. Antimitochondrial antibody negative primary biliary cirrhosis: a distinct syndrome of autoimmune cholangitis. Gut 1994:35:260-5.

10 Goodman ZD, McNally PR, Davis DR, et al. Autoimmune cholangitis: a variant of primary biliary cirrhosis. Clinicopathologic and serologic correlations in 200 cases. Dig Dis Sci 1995; 40:1232-42.

11 Kim WR, Poterucha JJ, Jorgensen RA, et al. Does antimitochondrial antibody status affect response to treatment in patients with primary biliary cirrhosis? Outcomes of ursodeoxycholic acid therapy and liver transplantation. Hepatology 1997;26:22-6.
12 Güldütuna S, You T, Kurtz W, et al. High performance liquid chromatographic determination of free and conjugated bile acids in serum, liver biopsies, bile, gastric juice and feces by fluorescence labeling. Clin Chim Acta 1993;214: 195-207

13 Terasaki PI, McClelland. Microdroplet assay for human serum lymphotoxins. Nature 1964;204:998-1000.

14 Bein G, Gläser R, Kirchner H. Rapid HLA-DRB1 genotyping by nested PCR amplication. Tissue Antigens 1992;39: 68-73.

15 Miller SA, Dykes DD, Polesky HF. A simple salting out procedure for extracting DNA from human nucleated cells. Nucleids Acids Res 1988;16:1215.

16 Ludwig J, Dickson ER, McDonald GSA. Staging of chronic nonsuppurative cholangitis (syndrome of primary biliary cirrhosis). Virchows Arch 1978;379:103-12.

17 Bengtsson BO, Thomson G. Measuring the strength of association between HLA antigens and diseases. Tissue Antigens 1981;18:356-63.

18 Svejgaard A, Jersild C, Staub Nielsen L, et al. HLA Antigens and disease: statistical and genetical considerations. Tissue Antigens 1974;4:95-105.

19 Jorgensen RA, Dickson ER, Hofmann AF, et al. Characterization of patients with a complete biochemical response to ursodeoxycholic acid. Gut 1995:36:935-8.

20 Bounand A-M, Heathcote EJ, Lindor KD, et al. Clinical significance of serum bilirubin levels under ursodeoxycholic acid therapy in patients with primary biliary cirrhosis. Hepatology 1999;29:39-43.

21 Angelopoulou K, Diamandis EP, Sutherland DJA, et al. Prevalence of serum antibodies against the p53 tumor suppressor gene protein in various cancers. Int f Cancer 1994; 58:480-7.

22 Livni N, Eid A, Ilan Y, et al. p53 expression in patients with cirrhosis with and without hepatocellular carcinoma. Cancer 1995;75:2420-6.

23 Klöppel G, Kirchhof M, Berg PA. Natural course of PBC. I. A morphological, clinical and serological analysis of 103 A morphological, clinical

24 Klein R, Pointer H, Zilly W, et al. Antimitochondrial antibody profiles in primary biliary cirrhosis distinguish at early stages between a benign and a progressive course: a prospective study on 200 patients followed for 10 years. Liver 1997;17:119-28

25 Dickson ER, Grambsch PM, Fleming TR, et al. Prognosis in primary biliary cirrhosis: model for decision making. Hepatology 1989;10:1-7.

26 Kilmurry MR, Heathcote EJ, Cauch-Dudek K, et al. Is the Mayo model for predicting survival useful after the introduction of ursodeoxycholic acid treatment for primary biliary cirrhosis? Hepatology 1996;23:1148-53.

27 Christensen E, Neuberger J, Crowe J, et al. Beneficial effect of azathioprine and prediction of prognosis in primary bilary cirrhosis: final results of an international trial. Gastroenterology 1985;89:1084-91.

28 Güldütuna S, Zimmer G, Imhof M, et al. Molecular aspects of membrane stabilization by ursodeoxycholate. Gastroenterology 1993;104:1736-44.

29 Güldütuna S, Deisinger B, Weiss A, et al. Ursodeoxycholic acid stabilizes phospholipid-rich membranes and mimics acid stabilizes phospholipid-rich membranes and mimics the effect of cholesterol: investigations on large uniles. Biochim Biophys Acta 1997;1326:265-74.

30 Beuers U, Throckmorton DC, Anderson MS, et al. Tauroursodeoxycholic acid activates protein kinase C in isolated rat hepatocytes. Gastroenterology 1996;110:155363.

31 Krähenbühl S, Fischer S, Talos C, et al. Ursodeoxycholate protects oxidative mitochondrial metabolism from bile acid toxicity: dose-response study in isolated rat liver mitochondria. Hepatology 1994;20:1595-601.

32 Krähenbühl S, Talos C, Fischer S, et al. Toxicity of bile acids on the electron transport chain of isolated rat liver mitochondria. Hepatology 1994;19:471-9.

33 Ljubuncic P, Fuhrman B, Oiknine J, et al. Effect of deoxycholic acid and ursodeoxycholic acid on lipid peroxydation in cultured macrophages. Gut 1996;39:475-8.

34 Setchell KDR, Rodriguez CMP, Clerici C. Bile acid concentrations in human and rat liver tissue and in hepatocyte nuclei. Gastroenterology 1997;112:226-35.

35 Ricci P, Hofmann AF, Hagey LR, et al. Adjuvant cholylsarcosine during ursodeoxycholic acid treatment of primary biliary cirrhosis. Dig Dis Sci 1998;43:1292-5. 